\title{
COMPLEMENT OF THE ZERO DIVISOR GRAPH OF A LATTICE
}

\author{
VINAYAK JOSHI ${ }^{\bowtie}$ and ANAGHA KHISTE
}

(Received 14 December 2012; accepted 4 January 2013; first published online 11 June 2013)

\begin{abstract}
In this paper, we determine when $\left(\Gamma_{I}(L)\right)^{c}$, the complement of the zero divisor graph $\Gamma_{I}(L)$ with respect to a semiprime ideal $I$ of a lattice $L$, is connected and also determine its diameter, radius, centre and girth. Further, a form of Beck's conjecture is proved for $\Gamma_{I}(L)$ when $\omega\left(\left(\Gamma_{I}(L)\right)^{c}\right)<\infty$.

2010 Mathematics subject classification: primary 05C15; secondary 05C40.

Keywords and phrases: zero divisor graph, complement of the graph, Beck's conjecture, $N$-prime ideal, $B$-prime ideal, semiprime ideal, diameter, radius, centre, girth.
\end{abstract}

\section{Introduction}

The investigation of graphs associated with various algebraic structures is a very important topic, well known and established in modern algebra. The graphs have played crucial roles in the study of ring constructions and their applications in coding theory (see Alfaro and Kelarev [2,3] and Bereg et al. [6]) and automata theory (see [16-18]), and in the study of commutative rings and semigroups (see [1, 4, 5, 19, 22, 23]).

The idea of associating a graph with the zero divisors of a commutative ring was introduced by Beck [5]. He was mainly interested in the colouring of such graphs. In recent years, zero divisor graphs have also been extensively studied by many mathematicians for ordered structures; see [8, 11-15, 20].

In this paper, we determine when $\left(\Gamma_{I}(L)\right)^{c}$, the complement of the zero divisor graph $\Gamma_{I}(L)$ with respect to a semiprime ideal $I$ of a lattice $L$, is connected and also determine its diameter, radius, centre and girth. Further, a form of Beck's conjecture is proved for $\Gamma_{I}(L)$ when $\omega\left(\left(\Gamma_{I}(L)\right)^{c}\right)<\infty$.

We begin with the necessary definitions.

A nonempty subset $I$ of a lattice $L$ is called a semi-ideal if for $a \in I, b \in L$, $b \leq a$ implies $b \in I$. A semi-ideal $I$ of a lattice $L$ is called an ideal of $L$ if $a, b \in I$

The first author gratefully acknowledges the financial supported by Board of College and University Development, University of Pune, via project-2012. The second author gratefully acknowledges the financial assistance in the form of CSIR Jr. Research Fellowship.

(C) 2013 Australian Mathematical Publishing Association Inc. 0004-9727/2013 \$16.00 
implies $a \vee b \in I$. Dually, we have the concepts of semi-filter and filter. An ideal $I$ of $L$ is said to be principal ideal generated by $a \in L$ if $I=(a]=\{x \mid x \leq a\}$. Dually, we have the concept of principal filter generated by $a$. A proper semi-ideal (ideal) $I$ of a lattice $L$ is called prime if $a, b \in L$ and $a \wedge b \in I$ imply $a \in I$ or $b \in I$. An ideal $I$ of a lattice $L$ is said to be semiprime if $a \wedge b \in I$ and $a \wedge c \in I$ together imply that $a \wedge(b \vee c) \in I$. Dually, we have the concept of semiprime filter and prime filter. A prime ideal $P$ of a lattice $L$ is said to be minimal prime ideal belonging to an ideal $I$ if $I \subseteq P$ and there exists no prime ideal $Q$ such that $I \varsubsetneqq Q \varsubsetneqq P$.

For an ideal $I$ and a nonempty subset $A$ of a lattice $L$, define a subset $I: A$ of $L$ as follows: $I: A=\{z \in L \mid z \wedge a \in I, \forall a \in A\}$. If $A=\{x\}$ then we write $I: x$ instead of $I:\{x\}$. Note that, if $x \leq y$ for $x, y \in L$, then $I: y \subseteq I: x$. Observe that $I \subseteq I: A$ and $I: A=\bigcap_{x \in A} I: x$. Note that $I: A$ need not be an ideal but it is a semi-ideal. Further, if $I$ is a semiprime ideal of $L$, then $I: A$ is an ideal of $L$. Moreover, if $I=(0]$ then $I: x$ is nothing but $\operatorname{Ann}(x)=\{y \mid y \wedge x=0\}$.

Let $L$ be a lattice and let $I$ be an ideal in $L$. Denote by $Z_{I}(L)=\{x \in L \mid x \wedge y \in I$ for some $y \in L \backslash I\}$ and $Z_{I}(L)^{*}=\{x \in L \backslash I \mid x \wedge y \in I$ for some $y \in L \backslash I\}$. Clearly, $Z_{I}(L)^{*} \cup I=Z_{I}(L)$.

Heinzer and Ohm [10] introduced the concept of $N$-prime (Nagata prime) ideals in commutative rings. We extend this concept to lattices.

Let $I$ be an ideal of a lattice $L$. A prime ideal $P$ of a lattice $L$ is said to be a maximal $N$-prime of $I$ if $P$ is maximal with respect to the property of being contained in $Z_{I}(L)=Z_{I}(L)^{*} \cup I$.

A prime ideal $P$ of a lattice $L$ is said to be a $B$-prime of $I$ (Bourbaki prime) if $P=I: x$ for some $x \in L$. A $B$-prime ideal of $I$ is also known as an associated prime ideal of $I$. More details on $B$-prime ideals in rings can be found in [7].

Rav's Theorem (Rav [21]). Let L be a lattice, I be an ideal of $L$ such that $I \cap F=\emptyset$ for a semiprime filter $F$ in $L$. Then there exists a prime ideal $P \supseteq I$ and $P \cap F=\emptyset$.

LEMMA 1.1 (Rav [21]). Every semiprime ideal is representable as an intersection of prime ideals.

Remark 1.2. Let $I$ be a semiprime ideal of a lattice $L$ with 1 . Let $S=L \backslash Z_{I}(L)$. Clearly, $S$ is a nonempty subset of $L$. We prove that $S$ is a semiprime filter of $L$. Let $a, b \in S$. Suppose, to the contrary, that $a \wedge b \notin S$, that is, $a \wedge b \in Z_{I}(L)$. Then there exists $c \notin I$ such that $a \wedge b \wedge c \in I$. Clearly, $b \wedge c \in I$, otherwise $a \in Z_{I}(L)$ which is not possible. Hence, $b \wedge c \in I$ together with $c \notin I$ gives $b \in Z_{I}(L)$, again a contradiction. Thus we have $a \wedge b \in S$. Now let $a \in S$ and $b \geq a$. Suppose $b \notin S$, that is, $b \in Z_{I}(L)$. Since $Z_{I}(L)$ is a semi-ideal, $a \in Z_{I}(L)$. This contradicts the fact that $a \in S$. This proves that $S$ is a filter of $L$.

Now we prove that $S$ is semiprime. Let $a \vee b, a \vee c \in S$. We claim that $a \vee(b \wedge c) \in S$. Suppose on the contrary that $a \vee(b \wedge c) \notin S$. Then $a \vee(b \wedge c) \in Z_{I}(L)$. Thus there exists $d \notin I$ such that $(a \vee(b \wedge c)) \wedge d \in I$. Hence $a \wedge d \in I$ and $b \wedge c \wedge d \in I$. If $b \wedge d \in I$, then using semiprimeness of $I$, we have $(a \vee b) \wedge d \in I$. Therefore, $a \vee b \in Z_{I}(L)$, 
a contradiction. Thus $b \wedge d \notin I$. Now again $a \wedge(b \wedge d) \in I$ and $c \wedge(b \wedge d) \in I$ with $I$ a semiprime ideal of $L$, so we have $(a \vee c) \wedge(b \wedge d) \in I$. This gives $a \vee c \in Z_{I}(L)$, again a contradiction. Hence $a \vee(b \wedge c) \in S$. Thus $S$ is a semiprime filter of $L$.

Let $x \in Z_{I}(L)$. Then $(x] \cap S=\emptyset$. Therefore, by Rav's theorem, there exists a prime ideal $P$ in $L$ such that $(x] \subseteq P$ and $P \cap S=\emptyset$. Clearly, $P \subseteq Z_{I}(L)$. Using Zorn's lemma, we can obtain a maximal $N$-prime $P_{1}$ of $I$ such that $x \in P_{1}$. Thus, for every $x \in Z_{I}(L)$ there exists a maximal $N$-prime $P_{i}$ of $I$ such that $x \in P_{i}$. Therefore, $Z_{I}(L) \subseteq \bigcup_{i \in \Lambda} P_{i}$. By the definition of maximal $N$-prime of $I$, each $P_{i} \subseteq Z_{I}(L)$ and hence $\bigcup_{i \in \Lambda} P_{i} \subseteq Z_{I}(L)$. Thus, if $\left\{P_{i}\right\}_{i \in \Lambda}$ denotes the set of all maximal $N$-primes of $I$ in $L$, then $Z_{I}(L)=\bigcup_{i \in \Lambda} P_{i}$. Further, we observed that $I \subseteq \bigcap_{i \in \Lambda} P_{i}$. For this, if there is a maximal $N$-prime ideal $P_{1}$ of $I$ such that $I \nsubseteq P_{1}$, then there exists $x \in I$ such that $x \notin P_{1}$. We prove that $P_{1} \vee(x] \subseteq Z_{I}(L)$. Let $t$ be any element in $P_{1} \vee(x]$. Hence $t \leq p \vee x$ for some $p \in P_{1}$. As $P_{1} \subseteq Z_{I}(L)$, we have $p \in Z_{I}(L)$. Therefore, there exists $q \notin I$ such that $p \wedge q \in I$. Since $x \in I, x \wedge q \in I$. By semiprimeness of $I$, we have $(p \vee x) \wedge q \in I$ and hence $t \wedge q \in I$. This together with $q \notin I$ gives $t \in Z_{I}(L)$. Thus $P_{1} \vee(x] \subseteq Z_{I}(L)$. Now again $\left(P_{1} \vee(x]\right) \cap S=\emptyset$, for a semiprime filter $S=L \backslash Z_{I}(L)$. By Rav's theorem, there exists a prime ideal $Q$ in $L$ such that $P_{1} \vee(x] \subseteq Q$ and $Q \cap S=\emptyset$. Clearly, $Q \subseteq Z_{I}(L)$. Hence $P_{1} \varsubsetneqq P_{1} \vee(x] \subseteq Q \subseteq Z_{I}(L)$, a contradiction to the fact that $P_{1}$ is maximal $N$-prime ideal of $I$.

\section{The connectivity of $\left(\Gamma_{I}(L)\right)^{c}$}

Joshi [11] introduced the concept of the zero divisor graph of a poset $P$ having the smallest element 0 with respect to an ideal $I$ of $P$. We mention below this definition when the corresponding poset is a lattice.

Definition 2.1. Let $I$ be an ideal of a lattice $L$ with 0 . We associate an undirected graph, called the zero divisor graph of $L$ with respect to the ideal $I$, denoted by $\Gamma_{I}(L)$ in which the set of vertices is $V\left(\Gamma_{I}(L)\right)=Z_{I}(L)^{*}=\{x \in L \backslash I \mid x \wedge y \in I$ for some $y \in L \backslash I\}$ and two distinct vertices $x, y$ are adjacent if and only if $x \wedge y \in I$.

It is clear from the definition that if an ideal $I$ is prime then $Z_{I}(L)^{*}=\emptyset$. Throughout the paper, we assume that $I$ is a semiprime ideal of a bounded lattice $L$ and $Z_{I}(L)^{*}=$ $V\left(\Gamma_{I}(L)\right)$ is nonempty. Hence $\left|Z_{I}(L)^{*}\right| \geq 2$.

We recall the following concepts from graph theory.

Let $G=(V, E)$ be a simple graph. The complement of $G$, denoted by $G^{c}$, is defined by setting $V\left(G^{c}\right)=V(G)=V$ and two distinct vertices $u, v \in V$ are joined by an edge in $G^{c}$ if and only if there exists no edge in $G$ joining $u$ and $v$. Let $x, y \in V$ be distinct vertices. We denote by $d(x, y)$ the length of a shortest path from $x$ to $y$, if one exists, and put $d(x, y)=\infty$ if no such path exists. The diameter of $G$ is zero if $G$ is the graph on one vertex and is $\operatorname{diam}(G)=\sup \{d(x, y) \mid x, y \in V\}$ otherwise. For any $v \in V$, the eccentricity of $v$ denoted by $e(v)$ is defined as $e(v)=\sup \{d(u, v) \mid u \in V\}$. The set of vertices of $G$ with minimal eccentricity is called the centre of the graph and the minimum eccentric value is called the radius of $G$ and is denoted by $r(G)$. The girth 
(a)
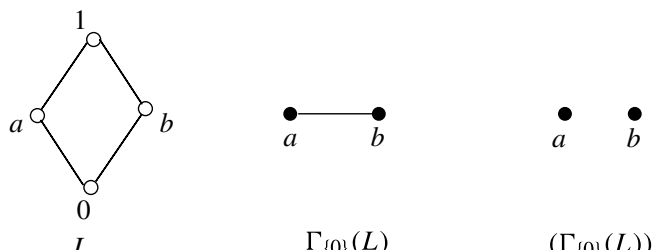

L

$\Gamma_{\{0\}}(L)$

$\left(\Gamma_{\{0\}}(L)\right)^{c}$

(b)

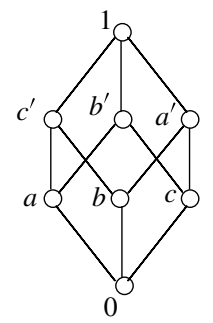

L

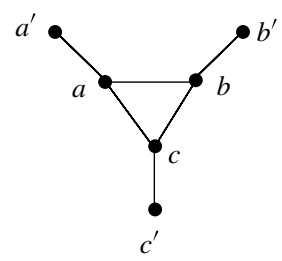

$\Gamma_{\{0\}}(L)$

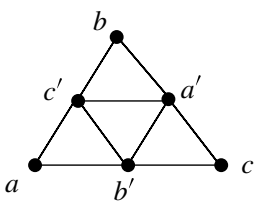

$\left(\Gamma_{\{0\}}(L)\right)^{c}$

FIGURE 1. (a) Connected zero divisor graph whose complement is disconnected. (b) A zero divisor graph and its complement both connected.

of the graph $G$, denoted by $\operatorname{gr}(G)$, is defined as the length of a shortest cycle in $G$. If $G$ does not contain any cycle, then we set $\operatorname{gr}(G)=\infty$. A graph $G$ is called hypertriangulated (triangulated) if each edge (vertex) of $G$ is an edge (vertex) of a triangle.

Undefined terms and notations are from Harary [9].

The following result is an easy consequence of Joshi [11, Theorem 2.4].

Theorem 2.2 (Joshi $[11])$. The zero divisor graph $\Gamma_{I}(L)$ is connected and diam $\left(\Gamma_{I}(L)\right)$ $\leq 3$.

From Figure 1(a), it is clear that $\Gamma_{\{0\}}(L)$ is connected but $\left(\Gamma_{\{0\}}(L)\right)^{c}$ is not, whereas in Figure 1(b), $\Gamma_{\{0\}}(L)$ and $\left(\Gamma_{\{0\}}(L)\right)^{c}$ both are connected. Hence it is natural to ask the following question.

Question. When is $\left(\Gamma_{I}(L)\right)^{c}$ connected?

We answer this question in the following theorem.

Main Theorem. The graph $\left(\Gamma_{I}(L)\right)^{c}$ is connected if and only if L does not have exactly two maximal $N$-primes $P_{1}, P_{2}$ of I such that $P_{1} \cap P_{2}=I$.

Furthermore, if $\left(\Gamma_{I}(L)\right)^{c}$ is connected then the following statements hold.

(1) $\operatorname{diam}\left(\left(\Gamma_{I}(L)\right)^{c}\right)=2$.

(2) $e(x)=2$ for all $x \in V\left(\left(\Gamma_{I}(L)\right)^{c}\right)$. Hence $r\left(\left(\Gamma_{I}(L)\right)^{c}\right)=2$ and the centre of $\left(\Gamma_{I}(L)\right)^{c}$ is $V\left(\left(\Gamma_{I}(L)\right)^{c}\right)$.

(3) $\left.\operatorname{gr}\left(\Gamma_{I}(L)\right)^{c}\right)=3$. In fact, $\left.\left(\Gamma_{I}(L)\right)^{c}\right)$ is hypertriangulated. 
We present the proof of the Main Theorem in three subsections, as follows:

(1) $L$ has exactly one maximal $N$-prime of $I$;

(2) $L$ has exactly two maximal $N$-primes of $I$;

(3) $L$ has at least three maximal $N$-primes of $I$.

We first prove the following two lemmas which will be used frequently in subsequent sections.

Lemma 2.3. If $\left(\Gamma_{I}(L)\right)^{c}$ is connected, then $\operatorname{diam}\left(\left(\Gamma_{I}(L)\right)^{c}\right) \geq 2$.

Proof. Let $a, b \in Z_{I}(L)^{*}, a \neq b$. By Theorem 2.2, $\Gamma_{I}(L)$ is connected; hence there exists $c \in Z_{I}(L)^{*}$ with $c \neq a$ such that $c \wedge a \in I$. Hence, if $\left(\Gamma_{I}(L)\right)^{c}$ is connected, then $d(a, c) \geq 2$ in $\left(\Gamma_{I}(L)\right)^{c}$ and so $\operatorname{diam}\left(\left(\Gamma_{I}(L)\right)^{c}\right) \geq 2$.

Lemma 2.4. Let $Q$ be a maximal $N$-prime of I in L. Let $x, y \in L \backslash I$ be any two elements such that $Q \subseteq I: x \cup I: y$. Then either $Q=I: x$ or $Q=I: y$.

Proof. Let $Q$ be a maximal $N$-prime of $I$ in $L$. Let $x, y \in L \backslash I$ be any two elements such that $Q \subseteq I: x \cup I: y$. Note that $I: x \cup I: y \subseteq Z_{I}(L)$. Let $S=L \backslash Z_{I}(L)$. Clearly, $I: x \cap S=\emptyset$ and $I: y \cap S=\emptyset$. As $I: x, I: y$ are ideals in the lattice $L$ and $S$ is the semiprime filter, by Rav's theorem, there exist prime ideals $Q_{1}$ and $Q_{2}$ such that $I: x \subseteq Q_{1}, I: y \subseteq Q_{2}$ and $Q_{1} \cap S=\emptyset=Q_{2} \cap S$. Since $Q \subseteq I: x \cup I: y$, then either $Q \subseteq I: x \subseteq Q_{1}$ or $Q \subseteq I: y \subseteq Q_{2}$. Further, $Q$ is a maximal $N$-prime of $I$ in $L$, which implies that either $Q=Q_{1}=I: x$ or $Q=Q_{2}=I: y$.

When $\mathbf{L}$ has exactly one maximal $\boldsymbol{N}$-prime of $\boldsymbol{I}$. Our aim in this subsection is to prove the following theorem.

THeOREM 2.5. If $P$ is the only maximal $N$-prime of I in $L$, then $\left(\Gamma_{I}(L)\right)^{c}$ is connected and $\operatorname{diam}\left(\Gamma_{I}(L)\right)^{c}=2$.

Proof. First, we claim that $P$ is not a $B$-prime of $I$. On the contrary, suppose that $P=I: a$ for some $a \in L$. If $a \in I$, then $L=I: a=P$, a contradiction. Hence $a \in L \backslash I$. By the hypothesis, $Z_{I}(L)=P$ gives $P \backslash I=Z_{I}(L)^{*}$ and $\left|Z_{I}(L)^{*}\right| \geq 2$, and there exists an element $(a \neq) b \in Z_{I}(L)^{*}=P \backslash I$. Then $a \in Z_{I}(L)^{*} \subseteq P=I: a$, a contradiction. Hence $P \neq I: x$ for any $x \in L \backslash I$, that is, $P$ is not a $B$-prime of $I$.

Let $a, b \in V\left(\left(\Gamma_{I}(L)\right)^{c}\right)$ be any two nonadjacent vertices in $\left(\Gamma_{I}(L)\right)^{c}$. Clearly, $P \neq I: a$ and $P \neq I: b$. By Lemma 2.4, $P \nsubseteq I: a \cup I: b$. Thus there exists $w \in P$ such that $w \notin I: a \cup I: b$. Since $P=Z_{I}(L)$, we have $w \in Z_{I}(L)^{*}=V\left(\left(\Gamma_{I}(L)\right)^{c}\right)$ such that $w \wedge a \notin I$ and $w \wedge b \notin I$. This gives that $a-w-b$ is a path in $\left(\Gamma_{I}(L)\right)^{c}$. Hence $\left(\Gamma_{I}(L)\right)^{c}$ is connected and this together with Lemma 2.3 gives $\operatorname{diam}\left(\Gamma_{I}(L)\right)^{c}=2$.

EXAMPLE 2.6. We now provide an example of a distributive lattice with exactly one maximal $N$-prime ideal of $I$. Let $\mathbb{N}$ be the set of natural numbers. Consider $L=F \cup\{\mathbb{N}\}$ where $F=\{X \subseteq \mathbb{N}|| X \mid<\infty\}$. Consider the semiprime ideal $(0]=\{\emptyset\}$ in $L$. Then $F=Z_{(0]}(L)$ and $F$ is the only maximal $N$-prime ideal of $L$ which is not a $B$-prime of $(0]$. 


\section{When $L$ has exactly two maximal $N$-primes of $I$}

Lemma 2.7. Let $L$ have exactly two maximal $N$-primes of $I$, say $P_{1}$ and $P_{2}$. If $P_{1} \cap P_{2} \neq I$ then there exist $a \in P_{1} \backslash P_{2}$ and $b \in P_{2} \backslash P_{1}$ such that $a \wedge b \notin I$.

Proof. Since $P_{1} \neq P_{2}$, there exist $a \in P_{1} \backslash P_{2}$ and $b \in P_{2} \backslash P_{1}$. Clearly, $a \vee b \notin P_{1} \cup$ $P_{2}=Z_{I}(L)$. Hence $I: a \vee b=I$. Suppose $a \wedge b \in I$. Clearly, $I \subseteq P_{1} \cap P_{2}$ follows from Remark 1.2. Let $x \in P_{1} \cap P_{2}$ with $x \notin I$. Since $I: a \vee b=I$, either $a \wedge x \notin I$ or $b \wedge x \notin I$. If $a \wedge x \notin I$, then the elements $a \in P_{1} \backslash P_{2}$ and $b \vee x \in P_{2} \backslash P_{1}$ satisfy our claim. Otherwise, the elements $a \vee x \in P_{1} \backslash P_{2}$ and $b \in P_{2} \backslash P_{1}$ will satisfy our claim.

Lemma 2.8. Let $P, Q$ be distinct $B$-prime ideals of $I$ in $L$ with $P=I: x$ and $Q=I: y$ for some $x, y \in L \backslash I$. Then $x \wedge y \in I$.

Proof. Suppose to the contrary that $x \wedge y \notin I$. Then $x \notin I: y$ and $y \notin I: x$. By the primeness of $I: x$ and $I: y$, we have $I: x \subseteq I: y$ and $I: y \subseteq I: x$, a contradiction.

The following theorem is essentially due to Joshi [11, Theorem 2.14].

THEOREM 2.9. The graph $\Gamma_{I}(L)$ is a complete bipartite graph if and only if there exist prime ideals $P_{1}$ and $P_{2}$ such that $I=P_{1} \cap P_{2}$.

Theorem 2.10. Let L have exactly two maximal $N$-primes of $I$, say $P_{1}$ and $P_{2}$. Then the following statements hold:

(1) $\quad\left(\Gamma_{I}(L)\right)^{c}$ is connected if and only if $P_{1} \cap P_{2} \neq I$;

(2) if $\left(\Gamma_{I}(L)\right)^{c}$ is connected then $\operatorname{diam}\left(\left(\Gamma_{I}(L)\right)^{c}\right)=2$.

Proof. (1) Let $\left(\Gamma_{I}(L)\right)^{c}$ be connected. Suppose $P_{1} \cap P_{2}=I$. By Theorem 2.9, $\Gamma_{I}(L)$ is a complete bipartite graph and hence $\left(\Gamma_{I}(L)\right)^{c}$ is disconnected, a contradiction.

Conversely, assume that $P_{1} \cap P_{2} \neq I$. There are only two possibilities: either both $P_{1}$ and $P_{2}$ are $B$-primes of $I$ or at least one of them is not a $B$-prime of $I$.

Case (1). Both $P_{1}$ and $P_{2}$ are $B$-primes of $I$. Hence $P_{1}=I: x$ and $P_{2}=I: y$ for some $x, y \in L \backslash I$. By Lemma 2.8, $x \wedge y \in I$ and hence $x, y \in Z_{I}(L)^{*}=P_{1} \cup P_{2} \backslash I$. Since $P_{1} \cap P_{2} \neq I$, there exists $t \in P_{1} \cap P_{2} \backslash I$. Thus $t \wedge x, t \wedge y \in I$ and by the semiprimeness of $I$, we have $t \wedge(x \vee y) \in I$. This gives $x \vee y \in Z_{I}(L)=P_{1} \cup P_{2}$. Hence $x \vee y \in P_{1}$ or $x \vee y \in P_{2}$, a contradiction. Thus this case is not possible.

Case (2). At least one of $P_{1}, P_{2}$ is not a $B$-prime of $I$. Without loss of generality, we may assume that $P_{1}$ is not a $B$-prime of $I$.

We claim that there exist $a, b \in V\left(\left(\Gamma_{I}(L)\right)^{c}\right)$ such that $a \wedge b \in I$. If $a \wedge b \notin I$ for any $a, b \in V\left(\left(\Gamma_{I}(L)\right)^{c}\right)$, then $\left(\Gamma_{I}(L)\right)^{c}$ is a complete graph, a contradiction to Lemma 2.3. Hence assume that $a \wedge b \in I$ for some $a, b \in V\left(\left(\Gamma_{I}(L)\right)^{c}\right)$. Clearly, $P_{1} \neq I: a$ and $P_{1} \neq I: b$. By Lemma 2.4, $P_{1} \nsubseteq I: a \cup I: b$. Thus there exists $t \in P_{1}$ such that $t \notin I: a \cup I: b$. Clearly, $t \in V\left(\left(\Gamma_{I}(L)\right)^{c}\right)$ such that $a-t-b$ is a path in $\left(\Gamma_{I}(L)\right)^{c}$. This gives that $d(a, b)=2$, for any two nonadjacent vertices $a, b \in V\left(\left(\Gamma_{I}(L)\right)^{c}\right)$ and hence $\left(\Gamma_{I}(L)\right)^{c}$ is connected. 
(2) Let $\left(\Gamma_{I}(L)\right)^{c}$ be connected. By Lemma 2.3 and by the first part of this theorem, it follows that $\operatorname{diam}\left(\left(\Gamma_{I}(L)\right)^{c}\right)=2$.

Theorem 2.11. Suppose L has exactly two maximal $N$-primes of $I$, say $P_{1}$ and $P_{2}$. Then the following statements are equivalent:

(1) $\left(\Gamma_{I}(L)\right)^{c}$ is connected;

(2) $P_{1} \cap P_{2} \neq I$;

(3) $\operatorname{diam}\left(\Gamma_{I}(L)\right)=3$.

Proof. (1) $\Leftrightarrow$ (2) follows from Theorem 2.10.

(2) $\Rightarrow$ (3): Let $P_{1} \cap P_{2} \neq I$. By Lemma 2.7, there exist $a \in P_{1} \backslash P_{2}$ and $b \in P_{2} \backslash P_{1}$ such that $a \wedge b \notin I$. It is easy to see that $a, b \in Z_{I}(L)^{*}$. Further, $I: a \vee b=I$. Clearly, $a-b$ is not an edge in $\Gamma_{I}(L)$, that is, $d(a, b) \neq 1$ in $\Gamma_{I}(L)$. If there exists $c \in Z_{I}(L)^{*}$ such that $a-c-b$ is a path in $\Gamma_{I}(L)$, then by the semiprimeness of $I$, we have $c \in I: a \vee b=I$, a contradiction. Thus $d(a, b) \neq 2$ in $\Gamma_{I}(L)$. By Theorem 2.2, $\Gamma_{I}(L)$ is connected and $\operatorname{diam}\left(\Gamma_{I}(L)\right) \leq 3$, so we have $\operatorname{diam}\left(\Gamma_{I}(L)\right)=3$.

(3) $\Rightarrow(2)$ : Assume that $\operatorname{diam}\left(\Gamma_{I}(L)\right)=3$. Therefore, there exists a path $a-b-c-d$ of length three in $\Gamma_{I}(L)$. Hence $a \wedge b, b \wedge c, c \wedge d \in I$ in $L$. Since $a \in Z_{I}(L)=P_{1} \cup P_{2}$, $a \in P_{1}$ or $a \in P_{2}$. If $a \in P_{1} \cap P_{2}$, then we are done. Suppose $a \notin P_{1}$ and since $a \wedge b \in I \subseteq P_{1} \cap P_{2}, b \in P_{1}$. If $b \in P_{2}$, then again we are done. Let $b \notin P_{2}$. Since $b \wedge c \in I \subseteq P_{1} \cap P_{2}, c \in P_{2}$. If $c \in P_{1}$, then again $P_{1} \cap P_{2} \neq I$. Let $c \notin P_{1}$. As $c \wedge d \in$ $I \subseteq P_{1} \cap P_{2}$, this gives $d \in P_{1}$. Thus $a \in P_{2}$ and $d \in P_{1}$ implies that $a \wedge d \in P_{1} \cap P_{2}$. Since $a \wedge d \notin I, P_{1} \cap P_{2} \neq I$.

We now provide examples of lattices having exactly two maximal $N$-primes of $I$.

Example 2.12. Let $L=\{0,1, a, b\}$ with $a \wedge b=0$ and $a \vee b=1$. Consider a semiprime ideal $(0]$ in $L$. Then $(a]=P_{1},(b]=P_{2}$ are the only maximal $N$-primes of $(0]$ and which are $B$-prime of (0] also and $P_{1} \cap P_{2}=(0]$. Note that in this case, $\left(\Gamma_{(0]}(L)\right)^{c}$ is not connected.

Example 2.13. Let $\mathbb{N}$ be the set of natural numbers. Let $L=\{X \subseteq \mathbb{N}|| X \mid<\infty\} \cup\{\mathbb{N}-$ $\{1\}\} \cup\{\mathbb{N}\}$. Consider a semiprime ideal $(0]=\{\emptyset\}$ in $L$. Further $P_{1}=(\mathbb{N}-\{1\}]$ and $P_{2}=$ $\{X \subseteq \mathbb{N}|| X \mid<\infty\}$ are the only maximal $N$-prime ideals of (0] such that $P_{1} \cap P_{2} \neq(0]$. Note that $P_{1}=(0]:\{1\}$ is a $B$-prime of (0], whereas $P_{2}$ is not a $B$-prime of (0]. From Theorem 2.10, it is clear that $\left(\Gamma_{(0]}(L)\right)^{c}$ is connected.

\section{When $L$ has at least three maximal $N$-primes of $I$.}

THeorem 2.14. Let L have at least three maximal $N$-primes of $I$. Then $\left(\Gamma_{I}(L)\right)^{c}$ is connected and $\operatorname{diam}\left(\left(\Gamma_{I}(L)\right)^{c}\right)=2$.

Proof. We claim that there exist $x, y \in V\left(\left(\Gamma_{I}(L)\right)^{c}\right)$ such that $x \wedge y \in I$. If $x \wedge y \notin I$ for any $x, y \in V\left(\left(\Gamma_{I}(L)\right)^{c}\right)$, then $\operatorname{diam}\left(\left(\Gamma_{I}(L)\right)^{c}\right)=1$, a contradiction to $\operatorname{diam}\left(\left(\Gamma_{I}(L)\right)^{c}\right) \geq 2$, by Lemma 2.3. Thus, we have $x \wedge y \in I$ for some $x, y \in V\left(\left(\Gamma_{I}(L)\right)^{c}\right)$. Then $x$ and $y$ are not adjacent in $\left(\Gamma_{I}(L)\right)^{c}$. Let $P_{1}$ be a maximal $N$-prime of $I$. If $P_{1} \nsubseteq I: x \cup I: y$, then there exists $w \in P_{1}$ such that $w \notin I: x \cup I: y$. It is easy to see that $w \in Z_{I}(L)^{*}$ 
such that $x-w-y$ is a path of length two in $\left(\Gamma_{I}(L)\right)^{c}$ between $x$ and $y$. In such a case, we are done. Hence, assume that $P_{1} \subseteq I: x \cup I: y$. By Lemma 2.4, either $P_{1}=I: x$ or $P_{1}=I: y$. Without loss of generality, we may assume that $P_{1}=I: x$. By the hypothesis, $L$ has at least three maximal $N$-primes of $I$. Let $P_{2}$ be a maximal $N$-prime of $I$ such that $P_{2} \neq P_{1}$. Again, if $P_{2} \nsubseteq I: x \cup I: y$, then we are done. So we assume that $P_{2} \subseteq I: x \cup I: y$, then again by Lemma 2.4, we obtain that $P_{2}=I: y$. Let $P_{3}$ be a maximal $N$-prime of $I$ such that $P_{3} \notin\left\{P_{1}, P_{2}\right\}$. Note that $P_{3}$ is not a subset of $P_{1} \cup P_{2}=I: x \cup I: y$. Hence, there exists $t \in P_{3}$ such that $t \wedge x \notin I$ and $t \wedge y \notin I$, that is, there exists a path $x-t-y$ of length two in $\left(\Gamma_{I}(L)\right)^{c}$ between $x$ and $y$. This proves that $\left(\Gamma_{I}(L)\right)^{c}$ is connected and $\operatorname{diam}\left(\left(\Gamma_{I}(L)\right)^{c}\right) \leq 2$. From Lemma 2.3, we have $\operatorname{diam}\left(\left(\Gamma_{I}(L)\right)^{c}\right)=2$.

Example 2.15. Let $X$ be a set such that $|X|=n, n \geq 3$. Consider $L=P(X)$, the power set of $X$ and the semiprime ideal $(0]=\{\emptyset\}$ in $L$. Then the lattice $L$ has at least three maximal $N$-primes of $(0]$.

Lemma 2.16. Let $G=(V, E)$ be a simple connected graph such that $G^{c}$ is connected. If $x$ is any element of $V$ with $|V| \geq 2$, then $e(x) \geq 2$ in $G^{c}$.

Proof. Let $x \in V$. Since $|V| \geq 2$ and $G$ is connected, there exists $y \in V$ such that $x-y$ is an edge in $G$. Hence $x-y$ is not an edge in $G^{c}$. Thus $d(x, y) \geq 2$ in $G^{c}$. Therefore, $e(x) \geq 2$ in $G^{c}$.

Proof of the Main Theorem. From Theorem 2.5, Theorem 2.10 and Theorem 2.14, it follows that $\left(\Gamma_{I}(L)\right)^{c}$ is connected and $\operatorname{diam}\left(\left(\Gamma_{I}(L)\right)^{c}\right)=2$ if and only if $L$ does not have exactly two maximal $N$-primes $P_{1}, P_{2}$ of $I$ such that $P_{1} \cap P_{2}=I$.

Now we claim that $r\left(\left(\Gamma_{I}(L)\right)^{c}\right)=2$. Since $\left(\Gamma_{I}(L)\right)^{c}$ is connected, it follows from Lemma 2.16 and $\operatorname{diam}\left(\Gamma_{I}(L)\right)^{c}=2$ that for any $x \in Z_{I}(L)^{*}, e(x)=2$ in $\left(\Gamma_{I}(L)\right)^{c}$, proving $r\left(\left(\Gamma_{I}(L)\right)^{c}\right)=2$. Hence, the centre of $\left(\Gamma_{I}(L)\right)^{c}$ is the set of all vertices of $\left(\Gamma_{I}(L)\right)^{c}$.

Now we prove that $\operatorname{gr}\left(\left(\Gamma_{I}(L)\right)^{c}\right)=3$. For this, let $x \in Z_{I}(L)^{*}$, as $Z_{I}(L)^{*} \neq \emptyset$. Hence, there exists $y \notin I$ such that $x \wedge y \in I$. Clearly, $x, y \in V\left(\left(\Gamma_{I}(L)\right)^{c}\right)$ such that $x$ is not adjacent to $y$ in $\left(\Gamma_{I}(L)\right)^{c}$. Since $\left(\Gamma_{I}(L)\right)^{c}$ is connected and diam $\left(\left(\Gamma_{I}(L)\right)^{c}\right)=2$, we have $d(x, y)=2$ in $\left(\Gamma_{I}(L)\right)^{c}$, that is, there exists $z \in V\left(\left(\Gamma_{I}(L)\right)^{c}\right)$ such that $x-z-y$ is a path of length two in $\left(\Gamma_{I}(L)\right)^{c}$. As $y \notin I$ and $x \wedge z \wedge y \in I$, this implies that $x \wedge z \in Z_{I}(L)^{*}$. If $x \wedge z \notin\{x, z\}$, then $x-x \wedge z-z-x$ is a cycle of length three in $\left(\Gamma_{I}(L)\right)^{c}$ and hence we are done. So we may assume that $x \wedge z \in\{x, z\}$. Without loss of generality, we assume that $x \wedge z=x$, that is, $x<z$. Since $z \in Z_{I}(L)^{*}$, there exists $t \notin I$ such that $t \wedge z \in I$. Clearly, $t \in V\left(\left(\Gamma_{I}(L)\right)^{c}\right) \backslash\{x, y, z\}$ such that $t \wedge x \leq t \wedge z \in I$. Hence $d(x, t) \neq 1$ in $\left(\Gamma_{I}(L)\right)^{c}$. Since $\left(\Gamma_{I}(L)\right)^{c}$ is connected, therefore $d(x, t)=2$ in $\left(\Gamma_{I}(L)\right)^{c}$. Then there exists $w \in V\left(\left(\Gamma_{I}(L)\right)^{c}\right)$ such that $x-w-t$ is a path of length two in $\left(\Gamma_{I}(L)\right)^{c}$. Hence $x \wedge w \notin I$ which yields $z \wedge w \notin I$. Thus $x-w-z-x$ is a cycle of length three in $\left(\Gamma_{I}(L)\right)^{c}$. Hence $\operatorname{gr}\left(\left(\Gamma_{I}(L)\right)^{c}\right)=3$.

Now, we show that $\left(\Gamma_{I}(L)\right)^{c}$ is hypertriangulated. Let $x-z$ be any edge in $\left(\Gamma_{I}(L)\right)^{c}$. Proceeding as above, it can be shown that there exists $w \in V\left(\left(\Gamma_{I}(L)\right)^{c}\right) \backslash\{x, z\}$ such 


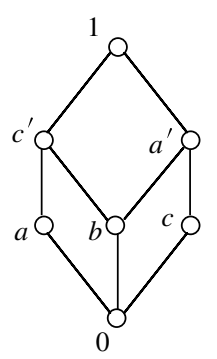

L

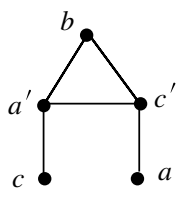

$\left(\Gamma_{\{0\}}(L)\right)^{c}$

FIGURE 2. A graph $\left(\Gamma_{\{0\}}(L)\right)^{c}$ with $\operatorname{diam}\left(\left(\Gamma_{\{0\}}(L)\right)^{c}\right)=3$.

that $w \wedge x, w \wedge z \notin I$. This shows that any edge of $\left(\Gamma_{I}(L)\right)^{c}$ is an edge of a triangle in $\left(\Gamma_{I}(L)\right)^{c}$. Thus $\left(\Gamma_{I}(L)\right)^{c}$ is hypertriangulated.

Remark 2.17. From the Main Theorem, it is clear that if $I$ is a semiprime ideal in $L$, then $\operatorname{diam}\left(\left(\Gamma_{I}(L)\right)^{c}\right)=2$. Note that if we drop the condition that $I$ is a semiprime ideal in $L$, then the assertion of the Main Theorem need not be true. Consider the lattice depicted in Figure 2. Then it is easy to see that $\operatorname{diam}\left(\left(\Gamma_{\{0\}}(L)\right)^{c}\right)=3$ for a nonsemiprime ideal (0].

Hence we raise the following question.

Question. Find the class $\mathcal{I}$ of ideals of a lattice $L$ such that for $I \in \mathcal{I}$, $\operatorname{diam}\left(\left(\Gamma_{I}(L)\right)^{c}\right)=3$.

From the Main Theorem, it is clear that $\left(\Gamma_{I}(L)\right)^{c}$ is connected and $\operatorname{gr}\left(\left(\Gamma_{I}(L)\right)^{c}\right)=3$ whenever there exist exactly two maximal $N$-prime ideals $P_{1}, P_{2}$ such that $P_{1} \cap P_{2} \neq I$. Hence it is natural ask the following question.

Question. Is $\operatorname{gr}\left(\left(\Gamma_{I}(L)\right)^{c}\right)=3$ when $P_{1} \cap P_{2}=I$ ?

We answer this question in the following result.

Lemma 2.18. Suppose that $L$ has exactly two maximal $N$-primes of $I$, say $P_{1}$ and $P_{2}$. If $P_{1} \cap P_{2}=I$, then $\left(\Gamma_{I}(L)\right)^{c}$ contains a cycle if and only if either $\left|P_{1} \backslash P_{2}\right| \geq 3$ or $\left|P_{2} \backslash P_{1}\right| \geq 3$ if and only if $\operatorname{gr}\left(\left(\Gamma_{I}(L)\right)^{c}\right)=3$.

Proof. By the hypothesis, $P_{1}$ and $P_{2}$ are the only two maximal $N$-primes of $I$ in $L$. By Remark 1.2, it follows that $Z_{I}(L)=P_{1} \cup P_{2}$. By Theorem 2.9, it is clear that $\Gamma_{I}(L)$ is a complete bipartite graph with vertex set $V_{1}=P_{1} \backslash P_{2}$ and $V_{2}=P_{2} \backslash P_{1}$. Then it is clear that $\operatorname{gr}\left(\left(\Gamma_{I}(L)\right)^{c}\right)=3$ if and only if $\left|P_{1} \backslash P_{2}\right| \geq 3$ or $\left|P_{2} \backslash P_{1}\right| \geq 3$.

We close this section by proving a sufficient condition for $\operatorname{gr}\left(\left(\Gamma_{I}(L)\right)^{c}\right)=3$.

Lemma 2.19. If there exist distinct elements $a, b, c \in Z_{I}(L)^{*} \backslash P$ for some prime ideal $P$ belonging to $I$, then $\operatorname{gr}\left(\left(\Gamma_{I}(L)\right)^{c}\right)=3$. 
Proof. As $P$ is a prime ideal of $L$ belonging to $I$, that is, $I \subseteq P$ and $a, b, c \in Z_{I}(L)^{*} \backslash P$, we obtain that $a \wedge b, b \wedge c, c \wedge a \notin P$ and so $a \wedge b, b \wedge c, c \wedge a \in L \backslash I$. Hence, it follows that $a-b-c-a$ is a cycle of length three in $\left(\Gamma_{I}(L)\right)^{c}$. Thus $\operatorname{gr}\left(\left(\Gamma_{I}(L)\right)^{c}\right)=3$.

\section{Beck's conjecture for $\left(\Gamma_{I}(L)\right)^{c}$}

Beck [5] introduced the notion of colouring in a commutative ring $R$ as follows. Let $G$ be a simple graph whose vertices are the elements of $R$ and two vertices $x$ and $y$ are adjacent if $x y=0$. Then the graph $G$ is known as the zero divisor graph of $R$. The chromatic number of $G$ is denoted by $\chi(G)$. Thus, $\chi(G)$ is the minimum number of colours which can be assigned to the elements of $R$ such that adjacent elements receive different colours. A clique of a graph $G$ is a complete subgraph and the supremum of the sizes of cliques in $G$, denoted by $\omega(G)$, is called the clique number of $G$. We always have $\chi(G) \geq \omega(G)$. Beck [5] conjectured that $\chi(G)=\omega(G)$ but Anderson and Naseer [4] gave an example of a commutative local ring $R$ with 32 elements for which $\chi(G)>\omega(G)$.

A form of Beck's conjecture is proved for the zero divisor graph of a poset with 0 by Halaš and Jukl [8] and for the zero divisor graph of a poset having the smallest element 0 with respect to an ideal by Joshi [11] under the assumption that the corresponding zero divisor graph does not contain an infinite clique. Therefore, we raise the following question.

Question. Is Beck's conjecture true for the complement of the zero divisor graph of a poset $P$ ?

We answer this question partially in the following theorem.

Theorem 3.1. Let $L$ be a finite Boolean lattice such that $|L|=2^{n}, n \in \mathbb{N}$. Then $\omega\left(\left(\Gamma_{\{0\}}(L)\right)^{c}\right)=\chi\left(\left(\Gamma_{\{0\}}(L)\right)^{c}\right)=2^{n-1}-1$.

Proof. Consider a Boolean lattice $L=\mathbf{2}^{n}$ for some $n \in \mathbb{N}$. Clearly, $V\left(\left(\Gamma_{\{0\}}(L)\right)^{c}\right)=$ $L \backslash\{0,1\}$. Hence $\left|V\left(\left(\Gamma_{\{0\}}(L)\right)^{c}\right)\right|=2\left(2^{n-1}-1\right)$. If $p$ is an atom in $L$, then we observe that $C_{p}=\{x \neq 1 \mid x \geq p\}=[p) \backslash\{1\}$ forms a clique in $\left(\Gamma_{\{0\}}(L)\right)^{c}$. Further, if $y \in V\left(\left(\Gamma_{\{0\}}(L)\right)^{c}\right) \backslash C_{p}$, then $y \wedge p=0$. Hence $C_{p}$ is a maximal clique in $\left(\Gamma_{\{0\}}(L)\right)^{c}$. Thus $\omega\left(\left(\Gamma_{\{0\}}(L)\right)^{c}\right)=\left|C_{p}\right|=2^{n-1}-1$. Thus $V\left(\left(\Gamma_{\{0\}}(L)\right)^{c}\right)$ contains a complete subgraph $K_{2^{n-1}-1}$.

Now we claim that either $x$ or $x^{\prime}$ (the complement of $x$ ) is a member of $C_{p}$.

If $x, x^{\prime} \in V\left(\left(\Gamma_{\{0\}}(L)\right)^{c}\right) \backslash C_{p}$, then $p \wedge x=0=p \wedge x^{\prime}$. By the distributivity of $L$, $p \wedge\left(x \vee x^{\prime}\right)=0$. This gives $p=0$, a contradiction. Hence either $x$ or $x^{\prime}$ belong to $C_{p}$.

Hence, for colouring $\left(\Gamma_{\{0\}}(L)\right)^{c}$, we first assign the colours to the elements of $C_{p}$. Therefore, we need $2^{n-1}-1$ colours. Now for any element $y \notin C_{p}$, we have $y^{\prime} \in C_{p}$ and we assign the colour of $y^{\prime}$ to $y$. Thus $\omega\left(\left(\Gamma_{\{0\}}(L)\right)^{c}\right)=\chi\left(\left(\Gamma_{\{0\}}(L)\right)^{c}\right)=\left|C_{p}\right|=2^{n-1}-1$. 
The following theorem is essentially due to Joshi [11].

Theorem 3.2 (Joshi [11]). If $\omega\left(\Gamma_{I}(L)\right)$ is finite, then L has only a finite number of minimal prime semi-ideals belonging to $I$ and if $n$ is this number, then $\chi\left(\Gamma_{I}(L)\right)=$ $\omega\left(\Gamma_{I}(L)\right)=n$.

In the following, we prove a form of Beck's conjecture (Theorem 3.3) for zero divisor graphs with respect to an ideal $I$ under the assumption that the complement of the corresponding zero divisor graph with respect to an ideal $I$ does not contain an infinite clique. Explicitly, we prove the following result.

THEOREM 3.3. If $\omega\left(\left(\Gamma_{I}(L)\right)^{c}\right)$ is finite, then L has only a finite number of minimal prime ideals belonging to $I$ and if $n$ is this number, then $\chi\left(\Gamma_{I}(L)\right)=\omega\left(\Gamma_{I}(L)\right)=n$.

First, we give bounds for the clique number of $\left(\Gamma_{I}(L)\right)^{c}$.

Lemma 3.4. Let $P$ be any prime ideal of $L$ belonging to $I$ (that is, $I \subseteq P$ ). Then the following statements hold.

(1) If $\left(\Gamma_{I}(L)\right)^{c}$ does not contain any infinite clique, then $Z_{I}(L) \backslash P$ is finite.

(2) If $\omega\left(\left(\Gamma_{I}(L)\right)^{c}\right)$ is finite, then $Z_{I}(L) \backslash P$ is finite and indeed $\left|Z_{I}(L) \backslash P\right| \leq$ $\omega\left(\left(\Gamma_{I}(L)\right)^{c}\right)$.

Proof. (1) Let $Z_{I}(L) \backslash P$ be an infinite set. We choose an infinite sequence of distinct elements $x_{i} \in Z_{I}(L) \backslash P$. Since $P$ is a prime ideal of $L$ belonging to $I$ and as $x_{i} \notin P$ for $i=1,2,3, \ldots$, it follows that $x_{i} \wedge x_{j} \notin I$ for all $i, j \in\{1,2,3, \ldots\}$. Observe that the subgraph of $\left(\Gamma_{I}(L)\right)^{c}$ induced on $\left\{x_{i} \mid i=1,2,3, \ldots\right\}$ is an infinite clique. This contradicts the assumption that $\left(\Gamma_{I}(L)^{c}\right)$ does not contain any infinite clique. Hence, $Z_{I}(L) \backslash P$ is finite.

(2) Let $\omega\left(\left(\Gamma_{I}(L)\right)^{c}\right)=n$. We assert that $\left|Z_{I}(L) \backslash P\right| \leq n$. Suppose to the contrary that $\left|Z_{I}(L) \backslash P\right| \geq n+1$. Let $\left\{x_{1}, x_{2}, \ldots, x_{n+1}\right\} \subseteq Z_{I}(L) \backslash P$. Then it is clear that the subgraph of $\left(\Gamma_{I}(L)\right)^{c}$ induced on $\left\{x_{1}, x_{2}, \ldots, x_{n+1}\right\}$ is a clique, a contradiction to $\omega\left(\left(\Gamma_{I}(L)\right)^{c}\right)=n$. Thus $\left|Z_{I}(L) \backslash P\right| \leq n=\omega\left(\left(\Gamma_{I}(L)\right)^{c}\right)$.

Lemma 3.5. Let $P$ be any prime ideal of $L$ belonging to $I$. Let $A=\{Q \mid Q$ is a prime ideal of L belonging to I such that $Q \subseteq Z_{I}(L)$ but $\left.Q \nsubseteq P\right\}$. Then the following statements hold.

(1) If $\left(\Gamma_{I}(L)\right)^{c}$ does not contain any infinite clique, then A can admit only a finite number of elements which are pairwise incomparable under inclusion.

(2) If $\omega\left(\left(\Gamma_{I}(L)\right)^{c}\right)$ is finite, then A can admit at most $\omega\left(\left(\Gamma_{I}(L)\right)^{c}\right)$ elements which are pairwise incomparable under inclusion.

Proof. (1) Suppose that $\left(\Gamma_{I}(L)\right)^{c}$ does not contain any infinite clique. Suppose on the contrary that there exist infinitely many elements in $A$ which are pairwise incomparable under inclusion. Hence there exist $Q_{i} \in A$ for $i=1,2,3, \ldots$ with $Q_{i}$ and $Q_{j}$ such that $Q_{i} \nsubseteq Q_{j}$ and $Q_{j} \nsubseteq Q_{i}$ for all $i, j \in\{1,2,3, \ldots\}$ with $i \neq j$. Now $Q_{i} \nsubseteq P$ for $i=$ $1,2,3, \ldots$ Hence there exists $x_{1} \in Q_{1} \backslash P$. Further, $Q_{2} \nsubseteq P \cup Q_{1}$, so there exist $x_{2} \in$ $Q_{2} \backslash\left(P \cup Q_{1}\right)$. Continuing in this way we get $x_{i} \in Q_{i} \backslash\left(P \cup Q_{1} \cup Q_{2} \cup \cdots \cup Q_{i-1}\right)$ 
for all $i=1,2,3, \ldots$ Observe that $\left\{x_{i} \mid i=1,2,3, \ldots\right\} \subseteq Z_{I}(L) \backslash P$. Hence $Z_{I}(L) \backslash P$ is infinite, a contradiction to Lemma 3.4(1).

(2) Let $\omega\left(\left(\Gamma_{I}(L)\right)^{c}\right)=n$. Suppose that $A$ admits more than $n$ elements which are pairwise incomparable under inclusion. Let $\left\{Q_{1}, \ldots, Q_{n+1}\right\} \subseteq A$ be such that $Q_{i}$ and $Q_{j}$ are not comparable for all $i, j \in\{1,2, \ldots, n+1\}$ with $i \neq j$. Let $x_{1} \in Q_{1} \backslash P$. As in (1), we can choose $x_{i} \in Q_{i} \backslash\left(P \cup Q_{1} \cup \cdots \cup Q_{i-1}\right)$ for $i=2, \ldots, n+1$. Observe that $\left\{x_{i} \mid\right.$ $i=1,2, \ldots, n+1\} \subseteq Z_{I}(L) \backslash P$. This implies that $\left|Z_{I}(L) \backslash P\right| \geq n+1>\omega\left(\left(\Gamma_{I}(L)\right)^{c}\right)$, a contradiction to Lemma 3.4(2).

LEMma 3.6. The following statements hold.

(1) If $\left(\Gamma_{I}(L)\right)^{c}$ does not contain any infinite clique, then (i) the set of maximal $N$ primes of I in L is finite, (ii) the set of minimal prime ideals of L belonging to I is finite.

(2) If $\omega\left(\left(\Gamma_{I}(L)\right)^{c}\right)$ is finite, then $(i) L$ can admit at most $\omega\left(\left(\Gamma_{I}(L)\right)^{c}\right)+1$ maximal $N$ primes of $I$ and if $L$ admits exactly $k$ maximal $N$-primes of $I$ with $k \geq 3$, then $k \leq \omega\left(\left(\Gamma_{I}(L)\right)^{c}\right)$, (ii) $L$ can admit at most $\omega\left(\left(\Gamma_{I}(L)\right)^{c}\right)+1$ minimal prime ideals belonging to $I$, and if $k$ is the number of minimal prime ideals of L belonging to $I$ with $k \geq 3$, then $k \leq \omega\left(\left(\Gamma_{I}(L)\right)^{c}\right)$.

Proof. (1) Let $P$ be a maximal $N$-prime of $I$ in $L$. Let $A=\{Q \mid Q$ is a maximal $N$-prime of $I$ in $L$ and $Q \neq P$ \}. Since any maximal $N$-prime of $I$ in $L$ is a subset of $Z_{I}(L)$ and as distinct maximal $N$-primes of $I$ in $L$ are not comparable under inclusion, it follows from Lemma 3.5(1) that $A$ is finite. It is clear that the set of all maximal $N$-primes of $I$ in $L$ is $A \cup\{P\}$. Thus $L$ can admit only a finite number of maximal $N$-primes of $I$.

If $P$ is any minimal prime ideal of $L$ belonging to $I$, then $P \subseteq Z_{I}(L)$. Using similar arguments as above, it is easy to see that $L$ can admit only a finite number of minimal prime ideals of $L$ belonging to $I$.

(2) Let $P$ be any maximal $N$-prime of $I$ in $L$ and let $A=\{Q \mid Q$ is a maximal $N$-prime of $I$ in $L$ and $Q \neq P\}$. Further, assume that $\omega\left(\left(\Gamma_{I}(L)\right)^{c}\right)=n$. Then by Lemma 3.5(1), $|A| \leq n$ and, using the same arguments as in the proof of (1), the set of all maximal $N$-primes of $I$ in $L$ is $A \cup\{P\}$. Thus $L$ can admit at most $n+1$ maximal $N$-primes of $I$.

Suppose that $L$ admits exactly $k$ maximal $N$-primes of $I$ with $k \geq 3$. Let $\left\{P_{1}, P_{2}, \ldots, P_{k}\right\}$ be the set of all maximal $N$-primes of $I$. Then $Z_{I}(L)=\bigcup_{i=1}^{k} P_{i}$. It is easy to see that there exists $x_{i} \in P_{i} \backslash \bigcup_{j \in\{1,2, \ldots, k\} \backslash\{i\}} P_{j}$. Clearly, $x_{i} \neq x_{j}$, for all $i, j \in\{1,2, \ldots, k\}$ with $i \neq j$. Since $k \geq 3$, there exists at least one $t \in\{1,2, \ldots, k\}$ such that both $x_{i}$ and $x_{j}$ are not in $P_{t}$ and hence $x_{i} \wedge x_{j} \notin I$. Thus the subgraph of $\left(\Gamma_{I}(L)\right)^{c}$ induced on $\left\{x_{i} \mid i=1,2, \ldots, k\right\}$ is a clique and so $k \leq \omega\left(\left(\Gamma_{I}(L)\right)^{c}\right)$.

Using similar arguments as above and using Lemma 3.5(2), the statement about minimal prime ideals can be proved.

Proof of Theorem 3.3. It follows from Lemma 3.6 that $L$ has only a finite number of minimal prime ideals of $L$ belonging to $I$, say $P_{i}, 1 \leq i \leq n$. Since $I$ is a semiprime ideal in $L$, by Lemma 1.1, we have $I=\bigcap_{i=1}^{n} P_{i}$. Now we define a colouring $f$ on vertices of $\Gamma_{I}(L)$ as $f(x)=\min \left\{i \mid x \notin P_{i}\right\}$. If $x, y \in V\left(\Gamma_{I}(L)\right)$ such that $x$ and $y$ are adjacent in $\Gamma_{I}(L)$, then $x \wedge y \in I=\bigcap_{i=1}^{n} P_{i}$. Clearly, $x \notin P_{i}$ and $y \notin P_{j}$ for some minimal 
(a)

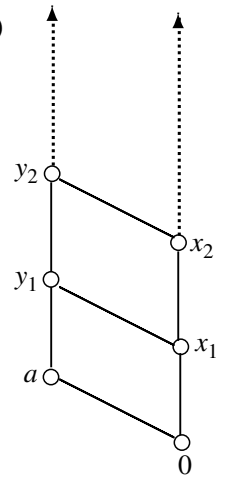

(b)

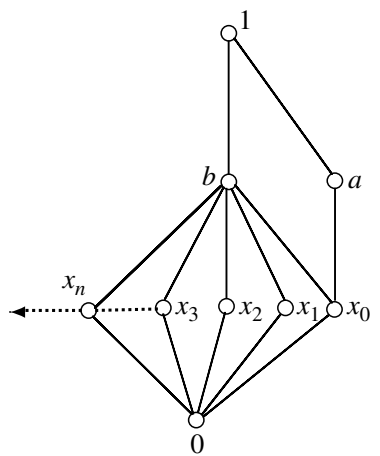

FigURE 3. (a): $\omega\left(\left(\Gamma_{I}(L)\right)\right)<\infty \nRightarrow \omega\left(\left(\Gamma_{I}(L)\right)^{c}\right)<\infty$ for semiprime ideal $I . \quad$ (b) $\omega\left(\left(\Gamma_{I}(L)\right)^{c}\right)<\infty \nRightarrow$ $\omega\left(\Gamma_{I}(L)\right)<\infty$ for non-semiprime ideal $I$.

prime ideals $P_{i}$ and $P_{j}$ belonging to $I$. Since $x \wedge y \in I=\bigcap_{i=1}^{n} P_{i}$, we conclude that $y \in P_{i}$ and $x \in P_{j}$. Thus $f(x) \neq f(y)$ and so $f$ is a colouring on $\Gamma_{I}(L)$. This implies $\chi\left(\Gamma_{I}(L)\right) \leq n$. Thus $\omega\left(\Gamma_{I}(L)\right) \leq \chi\left(\Gamma_{I}(L)\right) \leq n$. Hence $\omega\left(\Gamma_{I}(L)\right)$ is finite. Now we claim that, for a semiprime ideal $I$ of $L$ every minimal prime semi-ideal belonging to $I$ is a minimal prime ideal belonging to $I$. For this let $P$ be any minimal prime semiideal belonging to $I$. Let $x, y \in P$ be such that $x \vee y \notin P$ (such elements exist as $P$ is a semi-ideal). Clearly, $F=L \backslash P$ is a maximal filter with respect to the property that $I \cap F=\emptyset$. Thus $I \cap(F \vee[x)) \neq \emptyset$ and $I \cap(F \vee[y)) \neq \emptyset$. Let $u \in I \cap(F \vee[x))$ and $v \in I \cap(F \vee[y))$. Then $u \geq f_{1} \wedge x$ and $v \geq f_{2} \wedge y$ for some $f_{1}, f_{2} \in F$. Also, $u, v \in I$ give $\left(f_{1} \wedge f_{2}\right) \wedge x,\left(f_{1} \wedge f_{2}\right) \wedge y \in I$. By semiprimeness of $I$, we get $\left(f_{1} \wedge f_{2}\right) \wedge(x \vee y) \in I$. Using this fact and $\left(f_{1} \wedge f_{2}\right) \wedge(x \vee y) \in F$, we have $I \cap F \neq \emptyset$, a contradiction. Hence $P$ is a prime ideal belonging to $I$. Clearly, $P$ is a minimal prime ideal belonging to $I$. Thus, if $I$ is a semiprime ideal of $L$, then every minimal prime semi-ideal belonging to $I$ is a minimal prime ideal belonging to $I$. Hence, $L$ has only a finite number of minimal prime semi-ideals belonging to $I$ and if $n$ is this number, then this together with $\omega\left(\Gamma_{I}(L)\right)<\infty$ and Theorem 3.2 yields that $\chi\left(\Gamma_{I}(L)\right)=\omega\left(\Gamma_{I}(L)\right)=n$.

Remark 3.7. From Theorem 3.3, it is clear that for a semiprime ideal $I$ of a lattice $L$ with 0 , if $\omega\left(\left(\Gamma_{I}(L)\right)^{c}\right)<\infty$ then $\omega\left(\Gamma_{I}(L)\right)<\infty$. But the converse need not be true. Consider the lattice $L$ as shown in Figure 3(a). Then we observe that $\Gamma_{(0]}(L)$ has a finite clique while $\left(\Gamma_{(0]}(L)\right)^{c}$ does not. Also note that if we drop the condition that $I$ is a semiprime ideal in $L$ then the assertion that $\omega\left(\left(\Gamma_{I}(L)\right)^{c}\right)<\infty$ implies $\omega\left(\Gamma_{I}(L)\right)<\infty$ need not be true. Consider the lattice $L$ depicted in Figure 3(b). Clearly, (0] is not a semiprime ideal. Further it is easy to observe that $\left\{x_{0}, x_{1}, x_{2}, \ldots\right\}$ forms an infinite clique in $\Gamma_{(0]}(L)$ while $\left\{x_{0}, a\right\}$ is the only finite clique in $\left(\Gamma_{(0]}(L)\right)^{c}$.

\section{Acknowledgements}

The authors are grateful to the referees for their valuable suggestions. 


\section{References}

[1] M. Afkhami, M. Karimi and K. Khashyarmanesh, 'On the regular digraph of ideals of commutative rings', Bull. Aust. Math. Soc., doi:10.1017/S0004972712000792.

[2] R. Alfaro and A. V. Kelarev, 'Recent results on ring constructions for error-correcting codes, algebraic structures and their representations', Contemp. Math. 376 (2005), 1-12.

[3] R. Alfaro and A. V. Kelarev, 'On cyclic codes in incidence rings', Studia Sci. Math. Hungar. 43(1) (2006), 69-77.

[4] D. D. Anderson and M. Naseer, 'Beck's coloring of a commutative ring', J. Algebra 159 (1993), 500-514.

[5] I. Beck, 'Coloring of a commutative ring', J. Algebra 116 (1988), 208-226.

[6] S. Bereg, A. Kelarev and A. Salagean, 'Directed graphs and minimum distances of error-correcting codes in matrix rings', New Zealand J. Math. 33(2) (2004), 113-120.

[7] R. Gilmer and W. Heinzer, 'Ideals contracted from a Noetherian extension ring', J. Pure Appl. Algebra 24 (1982), 123-144.

[8] R. Halaš and M. Jukl, 'On Beck's coloring of posets', Discrete Math. 309 (2009), 4584-4589.

[9] F. Harary, Graph Theory (Narosa, New Delhi, 1988).

[10] W. Heinzer and J. Ohm, 'On the Noetherian-like rings of E. G. Evans', Proc. Amer. Math. Soc. 34(1) (1972), 73-74.

[11] V. V. Joshi, 'Zero divisor graph of a poset with respect to an ideal', Order 29 (2012), 499-506.

[12] V. V. Joshi and A. U. Khiste, 'On the zero divisor graph of a Boolean poset', Math. Slovaca, to appear.

[13] V. V. Joshi and A. U. Khiste, 'On the zero divisor graph of a pm-lattice', Discrete Math. 312 (2012), 2076-2082.

[14] V. V. Joshi, B. N. Waphare and H. Y. Pourali, 'Zero divisor graphs of lattices and primal ideals', Asian-Eur. J. Math. 5(3) (2012), 1250037 (9 pages).

[15] V. V. Joshi, B. N. Waphare and H. Y. Pourali, 'On generalized zero divisor graph of a poset', Discrete Appl. Math., doi:10.1016/j.dam.2012.12.019.

[16] A. V. Kelarev, Graph Algebras and Automata (Marcel Dekker, New York, 2003).

[17] A. V. Kelarev, 'Labelled Cayley graphs and minimal automata', Australas. J. Combin. 30 (2004), 95-101.

[18] A. V. Kelarev and D. S. Passman, 'A description of incidence rings of group automata', Contemp. Math. 456 (2008), 27-33.

[19] S. M. Moconja and Z. Z. Petrović, 'On the structure of comaximal graphs of commutative rings with identity', Bull. Aust. Math. Soc. 83(1) (2011), 11-21.

[20] S. K. Nimbhorkar, M. P. Wasadikar and L. DeMeyer, 'Coloring of meet-semilattices', Ars Combin. 84 (2007), 97-104.

[21] Y. Rav, 'Semiprime ideals in general lattices', J. Pure Appl. Algebra 56 (1989), 105-118.

[22] S. Visweswaran, 'Some results on the complement of the zero divisor graph of a commutative ring', J. Algebra Appl. 10(3) (2011), 573-595.

[23] S. Visweswaran, 'Some properties of the complement of the zero divisor graph of a commutative ring', ISRN Algebra (2011), 1-24; doi:10.5402/2011/591041.

VINAYAK JOSHI, Department of Mathematics, University of Pune,

Pune-411007, India

e-mail: vvj@math.unipune.ac.in

ANAGHA KHISTE, Department of Mathematics, University of Pune, Pune-411007, India

e-mail: avanikhiste@gmail.com 